\title{
Ovarian Cancer Metastasis to the Breast: A Case Report and Review of the Literature
}

\author{
Giuseppe Caruso $^{a} \quad$ Lucia Musacchio $^{a}$ Giusi Santangelo ${ }^{a}$ \\ Innocenza Palaia ${ }^{a}$ Federica Tomao ${ }^{a}$ Violante Di Donato ${ }^{a}$ \\ Giorgia Perniola ${ }^{a}$ Vanda Salutari ${ }^{b}$ Pierluigi Benedetti Panici ${ }^{a}$ \\ aDepartment of Maternal and Child Health and Urological Sciences, Sapienza University, \\ Policlinico Umberto I, Rome, Italy; 'bynecologic Oncology Unit, Department of Woman, \\ Child Health and Public Health, Fondazione Policlinico Universitario A. Gemelli, IRCCS, \\ Rome, Italy
}

\author{
Keywords \\ Ovarian cancer · Breast metastasis - Breast cancer - Chemotherapy
}

\begin{abstract}
Although ovarian cancer often presents as a widespread disease, metastases to the breast and/or axillary lymph nodes are a very rare event, accounting for only $0.03-0.6 \%$ of all breast cancers. Its early recognition and accurate distinction from primary breast cancer are of crucial importance to choose an adequate systemic therapy over unnecessary surgeries. We presented the case of a 53-year-old woman who was diagnosed with breast metastases 2 years after the diagnosis of advanced primary serous ovarian cancer. The patient underwent primary cytoreductive surgery and platinum-based chemotherapy in combination with bevacizumab, followed by bevacizumab maintenance for 18 months. After 2 years of negative follow-ups, the disease unexpectedly spread to the left breast and axillary lymph nodes. No axillary lymph node dissection or breast surgery was performed. The patient received axillary radiotherapy and multiple chemotherapy lines: gemcitabine/cisplatin, liposomal doxorubicin, topotecan, olaparib/cediranib, paclitaxel, and cisplatin. Unfortunately, none of these treatments improved her prognosis and she died 3 years after the disease recurrence. Ovarian cancer metastasis to the breast reveals a disseminated disease with a poor prognosis. Currently, no valid treatment options are available as the disease shows multidrug chemoresistance. In the era of precision medicine, the characterization of genetic and molecular markers may play a role in offering new promising targeted therapies.




\section{Background}

Epithelial ovarian cancer (EOC) is the primary cause of death from gynecologic cancers and the fifth most common cause of cancer mortality in women in developed countries [1]. Because an efficient screening program has not been developed to date, it often presents with a widely metastatic disease with $60-70 \%$ of patients diagnosed with International Federation of Gynecology and Obstetrics (FIGO) stage III or IV [2].

The typical course of metastatic ovarian cancer is intra-abdominal spread manifesting as peritoneal carcinomatosis, whereas the most common sites of distant metastases are pleura, liver, lung and lymph nodes. Metastasis of ovarian cancer to the breast (MOCB) and/or axillary lymph nodes is a rare event, accounting for only $0.03-0.6 \%$ of all breast neoplasms [3]. MOCB recognition and accurate distinction from primary breast cancer are of crucial importance for the patient management and prognosis, allowing in some instances to avoid unnecessary surgeries (e.g., mastectomy, lymphadenectomy) and choose an appropriate systemic chemotherapy [4]. Unfortunately, the diagnosis of MOCB is a clinical challenge as it presents with various clinical, radiological and pathological findings and may be misdiagnosed as a primary breast tumor [5].

Ovarian metastasis to the breast should be treated as a systemic disease with appropriate chemotherapeutic agents, whereas surgical management should be considered diagnostic and palliative [6]. The prognosis is generally poor as secondary breast cancer from ovarian cancer suggests concomitant disseminated disease.

In this paper, we describe the case of a woman with breast metastasis from ovarian cancer and present a brief review of the literature on this rare event.

\section{Case Presentation}

In 2016, a 53-year-old nulligravida woman presented to the Department of Maternal, Infant and Urological Sciences of Policlinico Umberto I (Sapienza University) in Rome for recurrent, advanced ovarian cancer with breast metastasis. Her clinical history started in October 2014 when she underwent primary cytoreductive surgery (total hysterectomy, bilateral salpingo-oophorectomy, omentectomy, pelvic and lomboaortic lymphadenectomy) for high-grade (G3), BRCA wild-type, serous EOC. From November 2014 to March 2015, she received 6 cycles of carboplatin (AUC5) and paclitaxel $\left(175 \mathrm{mg} / \mathrm{m}^{2}\right)$ i.v. every 21 days. Bevacizumab $\left(15 \mathrm{mg} / \mathrm{m}^{2}\right)$ was added during the last 2 cycles and then continued as maintenance therapy for 12 months up to April 2016.

Regularly scheduled follow-ups were negative until September 2016 when a total-body computed tomography (CT) scan revealed intraperitoneal spread (carcinosis), left axillary lymphadenopathy and a 3-cm-sized left breast mass in the upper outer quadrant. The patient had no family history of breast cancer and no history of previous breast pathology. The axillary lymph node and breast biopsies confirmed the diagnosis of recurrent, metastatic serous ovarian cancer, with a platinum-free interval of 18 months. The patient did not undergo any breast surgical treatment. From November 2016 to May 2017, she was submitted to 8 cycles of second-line chemotherapy with gemcitabine plus cisplatin (switched from carboplatin from the third cycle because of a hypersensitivity reaction with maculopapular erythematous rush over the neck and trunk). As the post-treatment imaging (positron emission tomography, PET/CT) showed persistence of breast disease, the patient underwent radiation therapy to the left axillary region ( 50 Gy in 25 fractions) from August to September 2017.

\section{Karger's}




\section{Case Reports in Oncology}

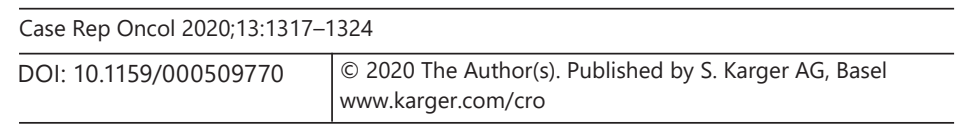

Caruso et al.: Ovarian Cancer Metastasis to the Breast
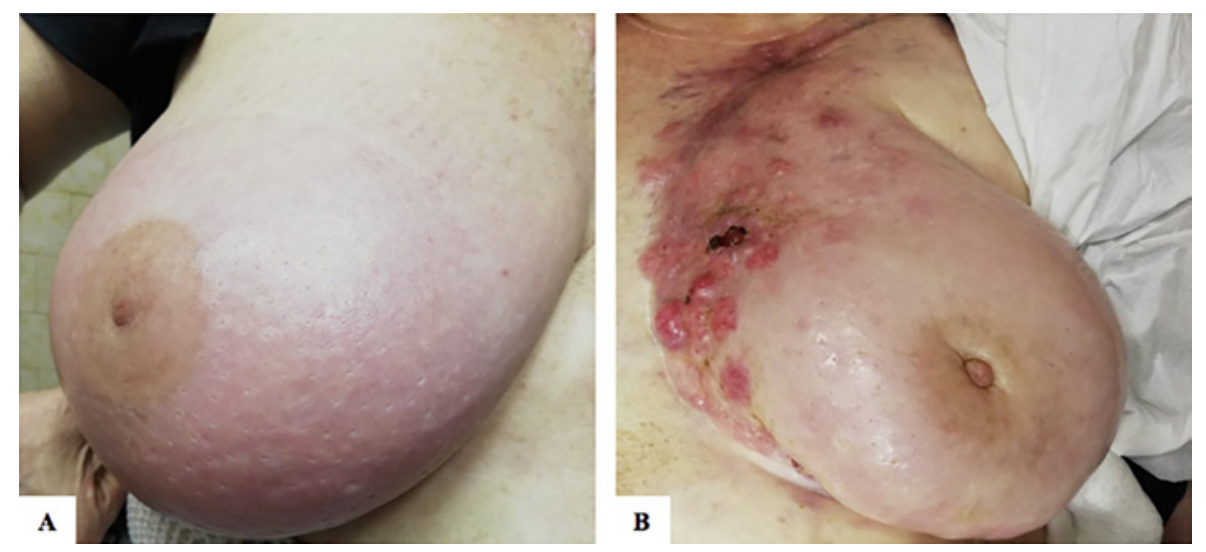

Fig. 1. A Initial breast inflammatory changes (erythema, edema, peau d'orange). B Breast necrosis and ulceration.

In November 2017, a new palpable breast lump appeared in the left breast and an additional biopsy was performed with the same histopathological results. Although an additional breast radiation treatment with a total dose of $35 \mathrm{~Gy}$ was performed, a disease progression to homolateral supraclavicular and internal mammary lymph nodes was reported; thus the patient underwent a third-line chemotherapy with 2 cycles of liposomal doxorubicin $(30 \mathrm{mg} /$ $\mathrm{m}^{2}$ ) from March to May 2018. The revaluation with PET/CT scan showed contralateral axillary lymphadenopathy and both neck and mediastinum involvement. Moreover, signs and symptoms of inflammatory and lymphangitic breast cancer appeared, including bilateral edema, erythema and peau d'orange (Fig. 1). From June to August 2018, a further line of chemotherapy consisting of 3 cycles of weekly topotecan was administered, again without any clinical benefit. Therefore, in October 2018, the patient was enrolled in the clinical trial BAROCCO [7], and she was randomized in the experimental arm with olaparib (300 $\mathrm{mg}$ twice daily, 7 days per week) plus cediranib (20 mg daily, 5 days per week). Even if the patient reported some clinical benefits, the total-body CT scan performed after 4 months of treatment revealed progression of disease and the patient abandoned the clinical trial.

Our last attempt was to administer weekly paclitaxel $\left(80 \mathrm{mg} / \mathrm{m}^{2}\right)$ and then weekly cisplatin $\left(20 \mathrm{mg} / \mathrm{m}^{2}\right)$. Unfortunately, in September 2019, the patient's general conditions appeared to be significantly compromised and she was referred to palliative care and hormonal therapy with letrozole, before dying a month later ( 5 years after ovarian cancer diagnosis and 3 years after the diagnosis of the breast metastasis).

\section{Discussion and Conclusions}

The present report describes a case of MOCB in a woman with invasive serous EOC. Ovarian cancer usually presents at an advanced stage, but the disease is confined within the peritoneal cavity in $85 \%$ of patients [2]. Distant metastases are unusual at presentation and during the course of the disease. In a study of 4,051 breast cancer women, Hajdu and Urban [8] found that the overall incidence of primary gynecologic tumor metastasis to the breast was $0.17 \%$, and only $0.07 \%$ of metastatic disease was related to primary ovarian carcinoma. However, significant advancements in ovarian cancer treatment may lead to an increased incidence of metastases in uncommon sites.

\section{Karger'k}




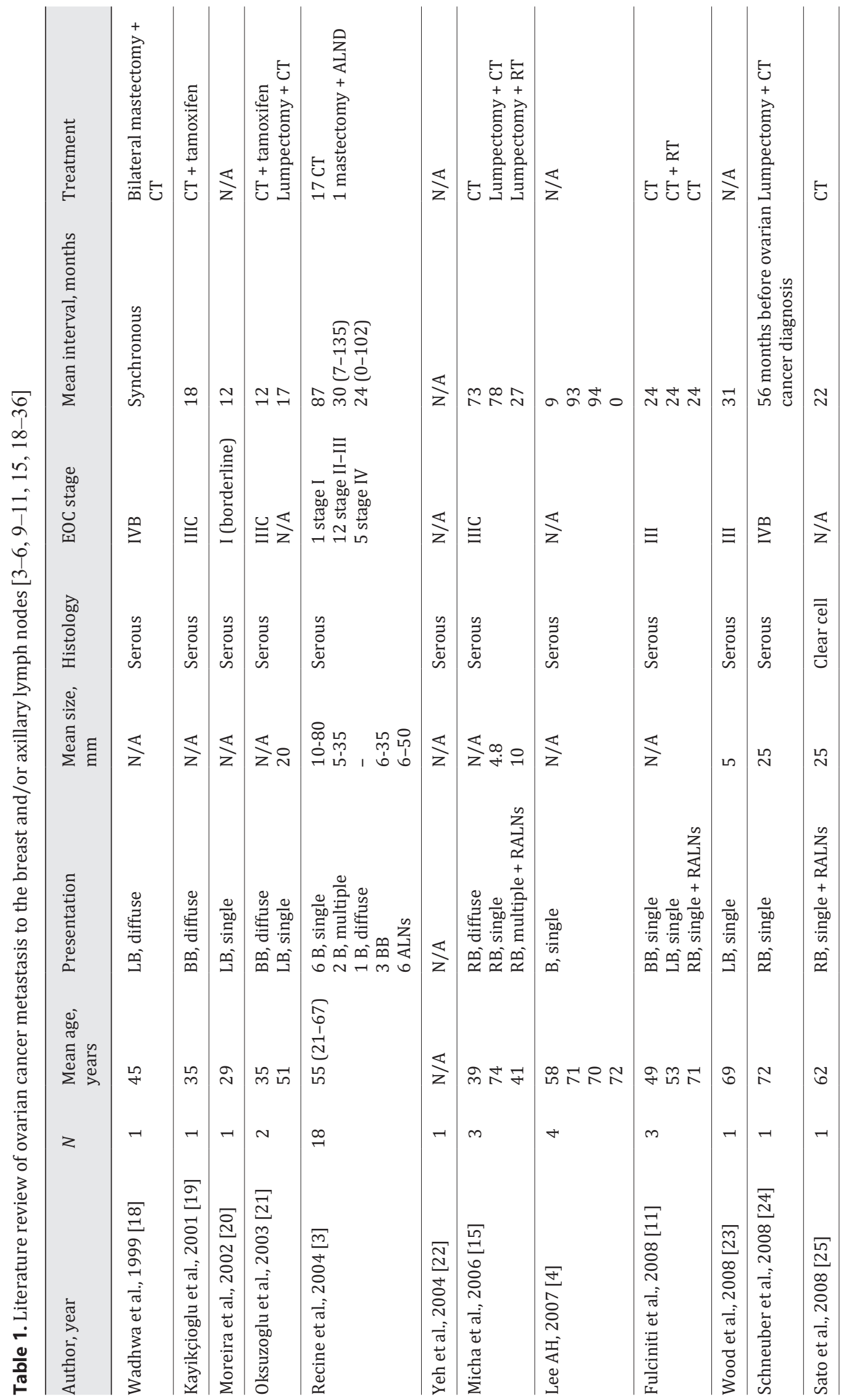




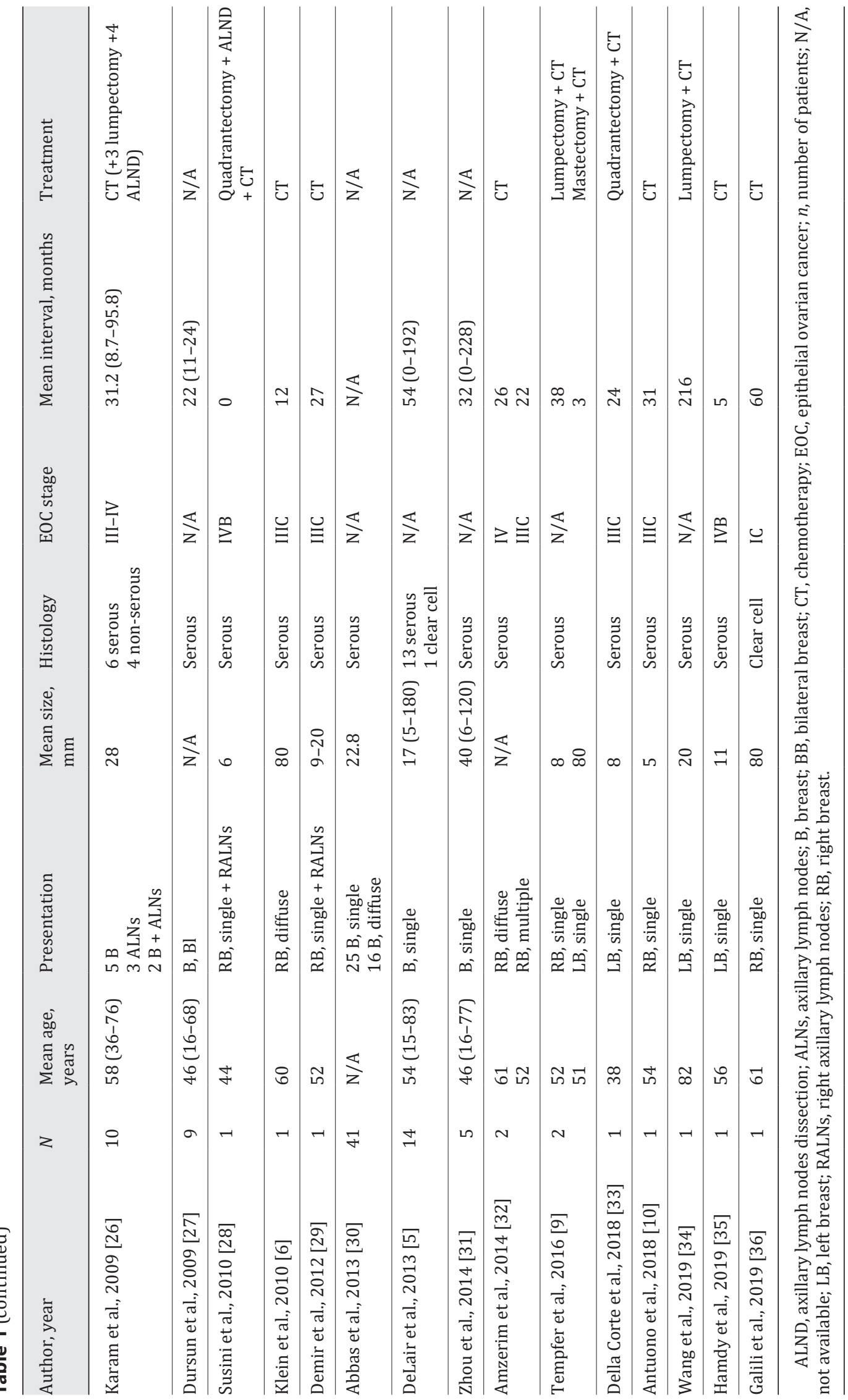


In 2016, Tempfer et al. [9] published a review of 110 cases of MOCB in advanced stage ovarian cancer patients with a poor prognosis. According to a Pubmed search performed in January 2020, using the search terms "breast metastasis", "ovarian cancer", "non-mammary breast metastasis" and "intramammary metastasis", we found more than 120 cases of MOCB reported in the literature, which are broadly summarized in Table 1.

Serous papillary carcinoma is the most common histotype of ovarian tumor associated with breast metastases [10], as it has been reported in $72 \%$ of the cases and it is usually detected within 2 years from the initial diagnosis of primary ovarian cancer [3], as in our case. Metastatic disease to the breast is more likely to present with an axillary mass, thus suggesting a lymphatic route of EOC spreading. Indeed, Lee [4] speculated that there may be some preferential lymphatic pathways including thoracic duct and left supraclavicular lymph nodes that could increase the risk of metastatic ovarian cancer to the left breast. It has been reported that the most common form of clinical presentation is a solitary mass, while only $4 \%$ of women have diffuse breast involvement [11]. Furthermore, the most frequent location is the upper outer quadrant in $62 \%$ of patients [12], as described in our case.

Breast and ovarian cancer share various environmental risk factors and hereditary gene mutations, which explains the increased risk of subsequent malignant breast tumors reported after ovarian cancer. Although MOCB is rare, it should be considered in the differential diagnosis of breast lesions in patients with a personal history of ovarian cancer. Indeed, the confirmation of diagnosis is of pivotal importance to choose an adequate therapeutic strategy and avoid unnecessary surgeries and overtreatment. However, unfortunately, due to the rarity and unspecific clinicopathological features of breast recurrences, it is difficult to make an accurate diagnosis, and a multidisciplinary approach is required to differentiate MOCB from a second primary breast cancer, especially since treatment and prognosis are quite different.

The optimal breast screening in women with a history of ovarian cancer should include breast self-exams, twice-yearly clinical breast exams, and annual mammography [13]. Among BRCA-mutated ovarian cancer patients without a personal history of breast cancer, preventive bilateral mastectomy or annual MRI screening is warranted only in early-stage ovarian cancer or if no recurrence has been detected for at least 10 years [14].

According to Micha et al. [15], ovarian metastasis to the breast should be treated as a systemic disease, with specific chemotherapeutic agents. Currently, surgical management for secondary breast cancer is considered diagnostic and reserved as a palliative measure, together with radiotherapy, for patients who are unresponsive to systemic chemotherapy agents. The survival times after breast metastatic disease secondary to ovarian cancer ranged from 13 days to 3.5 years, with a 1-year survival rate of about $40 \%$, as opposed to a 4 -year survival rate of $75 \%$ for patients with primary breast cancer [16]. MOCB reveals a widespread dissemination and the poor prognosis suggests that a selection of specific aggressive clones within the primary ovarian tumor could give rise to chemotherapy-resistant breast metastases. Indeed, high-grade serous ovarian cancer is characterized by genetic instability, which may be responsible for the development of a less indolent tumor biology with a remarkable impact on the metastatic tumor genome and treatment outcome [17].

In conclusion, MOCB represents a rare late-stage manifestation of ovarian cancer, which is associated with a dismal prognosis, regardless of the early diagnosis and treatment. Due to the disseminated nature of the disease, both surgery and radiotherapy find a place exclusively in palliative care. However, even the role of systemic chemotherapy appears limited as MOCB shows multidrug chemoresistance. Future characterization of the genetic and molecular markers of MOCB may play a role in offering new promising targeted therapies. 


\section{Case Reports in Oncology}

\begin{tabular}{l|l}
\hline Case Rep Oncol 2020;13:1317-1324 \\
\hline DOI: 10.1159/000509770 & $\begin{array}{l}\text { @ 2020 The Author(s). Published by S. Karger AG, Basel } \\
\text { www.karger.com/cro }\end{array}$ \\
\hline
\end{tabular}

Caruso et al.: Ovarian Cancer Metastasis to the Breast

\section{Acknowledgement}

We thank our colleagues and the staff of Policlinico Umberto I of Rome.

\section{Statement of Ethics}

The patient has given her written informed consent to publish her case (including publication of images).

\section{Conflict of Interest Statement}

The authors have no conflicts of interest to disclose.

\section{Funding Sources}

There are no funding sources.

\section{Author Contributions}

Concept and design: Giuseppe Caruso, Lucia Musacchio. Acquisition, analysis, or interpretation of data: Giuseppe Caruso, Lucia Musacchio, Giusi Santangelo. Drafting of the manuscript: Giuseppe Caruso, Lucia Musacchio, Giusi Santangelo. Critical revision of the manuscript for important intellectual content: Innocenza Palaia, Federica Tomao, Violante Di Donato, Giorgia Perniola, Vanda Salutari. Supervision: Innocenza Palaia, Pierluigi Benedetti Panici. All authors read and approved the final manuscript.

\section{References}

1 Bray F, Ferlay J, Soerjomataram I, Siegel RL, Torre LA, Jemal A. Global cancer statistics 2018: GLOBOCAN estimates of incidence and mortality worldwide for 36 cancers in 185 countries. CA Cancer J Clin. 2018;68(6): 394-424.

2 Heintz AP, Odicino F, Maisonneuve P, Quinn MA, Benedet JL, Creasman WT, et al. Carcinoma of the ovary. FIGO 26th Annual Report on the Results of Treatment in Gynecological Cancer. Int J Gynaecol Obstet. 2006;95(Suppl 1):S161-92.

3 Recine MA, Deavers MT, Middleton LP, Silva EG, Malpica A. Serous carcinoma of the ovary and peritoneum with metastases to the breast and axillary lymph nodes: a potential pitfall. Am J Surg Pathol. 2004;28(12): 1646-51.

4 Lee AH. The histological diagnosis of metastases to the breast from extramammary malignancies. J Clin Pathol. 2007;60(12):1333-41.

5 DeLair DF, Corben AD, Catalano JP, Vallejo CE, Brogi E, Tan LK. Non-mammary metastases to the breast and axilla: a study of 85 cases. Mod Pathol. 2013;26(3):343-9.

6 Klein RL, Brown AR, Gomez-Castro CM, Chambers SK, Cragun JM, Grasso-Lebeau L, et al. Ovarian cancer metastatic to the breast presenting as inflammatory breast cancer: a case report and literature review. J Cancer. 2010;1:27-31.

7 Colombo N, Nicoletto O, Benedetti Panici P, Tognon G, Lissoni AA, Bologna A, et al. BAROCCO: A randomized phase II study of weekly paclitaxel vs cediranib-olaparib with continuous schedule vs cediranib-olaparib with intermittent schedule in advanced platinum resistant ovarian cancer. Ann Oncol. 2018;29(Suppl 8):332-58.

8 Hajdu SI, Urban JA. Cancers metastatic to the breast. Cancer. 1972;29(6):1691-6.

9 Tempfer CB, El Fizazi N, Ergonenc H, Solass W. Metastasis of ovarian cancer to the breast: A report of two cases and a review of the literature. Oncol Lett. 2016;11(6):4008-12.

10 Antuono L, Angela F, Luca N, Giovanni M, Enrico C. Breast metastasis from ovarian cancer: A case report. Radiol Case Rep. 2018;13(6):1166-9.

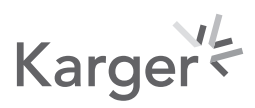




\section{Case Reports in Oncology}

11 Fulciniti F, Losito S, Botti G, Di Mattia D, La Mura A, Pisano C, et al. Metastases to the breast: role of fine needle cytology samples. Our experience with nine cases in 2 years. Ann Oncol. 2008;19(4):682-7.

12 Moore DH, Wilson DK, Hurteau JA, Look KY, Stehman FB, Sutton GP. Gynecologic cancers metastatic to the breast. J Am Coll Surg. 1998;187(2):178-81.

13 Committee on Practice Bulletins-Gynecology. Practice Bulletin Number 179: Breast Cancer Risk Assessment and Screening in Average-Risk Women. Obstet Gynecol. 2017;130(1):e1-e16.

14 McGee J, Giannakeas V, Karlan B, Lubinski J, Gronwald J, Rosen B, et al. Risk of breast cancer after a diagnosis of ovarian cancer in BRCA mutation carriers: Is preventive mastectomy warranted?. Gynecol Oncol. 2017; 145(2):346-51.

15 Micha JP, Goldstein BH, Epstein HD, Rettenmaier MA, Brown JV 3rd. Ovarian cancer metastatic to the breast. Gynecol Oncol. 2006;102(2):386-90.

16 Ozgüroğlu M, Ersavaşti G, Ilvan S, Hatemi G, Demir G, Demirelli FH. Bilateral inflammatory breast metastases of epithelial ovarian cancer. Am J Clin Oncol. 1999;22(4):408-10.

17 Wang ZC, Birkbak NJ, Culhane AC, Drapkin R, Fatima A, Tian R, et al. Profiles of genomic instability in highgrade serous ovarian cancer predict treatment outcome. Clin Cancer Res. 2012;18(20):5806-15.

18 Wadhwa J, Dawar R, Kumar L. Ovarian carcinoma metastatic to the breast. Clin Oncol (R Coll Radiol). 1999; 11(6):419-21.

19 Kayikçioğlu F, Boran N, Ayhan A, Güler N. Inflammatory breast metastases of ovarian cancer: a case report. Gynecol Oncol. 2001;83(3):613-6.

20 Moreira AL, Yao J, Waisman J, Cangiarella JF. Metastatic "borderline" papillary ovarian tumor in an intramammary lymph node. Breast J. 2002;8(5):309-10.

21 Oksuzoglu B, Abali H, Güler N, Beltali E, Ozisik. Metastasis to the breast from nonmammarian solid neoplasms: a report of five cases. Med Oncol. 2003;20:295-300.

22 Yeh CN, Lin CH, Chen MF. Clinical and ultrasonographic characteristics of breast metastases from extramammary malignancies. Am Surg. 2004;70(4):287-90.

23 Wood B, Sterrett G, Frost F, Swarbrick N. Diagnosis of extramammary malignancy metastatic to the breast by fine needle biopsy. Pathology. 2008;40(4):345-51.

24 Schneuber SE, Scholz HS, Regitnig P, Petru E, Winter R. Breast metastasis 56 months before the diagnosis of primary ovarian cancer: a case study. Anticancer Res. 2008;28(5B):3047-50.

25 Sato T, Muto I, Fushiki M, Hasegawa M, Hasegawa M, Sakai T, et al. Metastatic breast cancer from gastric and ovarian cancer, mimicking inflammatory breast cancer: report of two cases. Breast Cancer. 2008;15(4):31520.

26 Karam AK, Stempel M, Barakat RR, Morrow M, Gemignani ML. Patients with a history of epithelial ovarian cancer presenting with a breast and/or axillary mass. Gynecol Oncol. 2009;112(3):490-5.

27 Dursun P, Yanik FB, Kuscu E, Gultekin M, Ayhan A. Bilateral breast metastasis of ovarian carcinoma. Eur J Gynaecol Oncol. 2009;30(1):9-12.

28 Susini T, Olivieri S, Molino C, Castiglione F, Tavella K, Viligiardi R. Ovarian cancer initially presenting as intramammary metastases and mimicking a primary breast carcinoma: a case report and literature review. J Womens Health (Larchmt). 2010;19(1):169-74.

29 Demir L, Erten C, Yigit SC, Can A, Dirican A, Bayoglu V, et al. Intramammary lymph node metastasis in a patient with ovarian carcinoma and a brief review of the literature. Contemp Oncol (Pozn). 2012;16(2):108-10.

30 Abbas J, Wienke A, Spielmann RP, Bach AG, Surov A. Intramammary metastases: comparison of mammographic and ultrasound features. Eur J Radiol. 2013;82(9):1423-30.

31 Zhou S, Yu B, Cheng Y, Xu X, Shui R, Bi R, et al. Metastases to the breast from non-mammary malignancies: A clinicopathologic study of 28 cases. Zhonghua Bing Li Xue Za Zhi. 2014;43(4):231-5.

32 Amzerin M, Garcia C, Stanciu C, Veys I, Awada A, Errihani H, et al. Case Report: Mammary and rectal metastases from an ovarian cancer: report of two cases and review of literature. F1000Res. 2014;3:255.

33 Della Corte L, Giampaolino P, Fabozzi A, Cieri M, Zizolfi B, Morra I, et al. Breast metastasis two years after pelvic surgery and adjuvant chemotherapy for serous ovarian cancer. Gynecol Endocrinol. 2019;35(3):211-3.

34 Wang Z, Zhao D, Liu R, Zheng B. Ovarian cancer metastasis to the breast 18 years after the initial diagnosis: A case report. Medicine (Baltimore). 2019;98(43):e17577.

35 Hamdy 0, Shokeir FA, Saleh GA. Zaki MM Intramammary Nodal Metastasis from Ovarian Cancer: A Case Report. Eur J Breast Health. 2019;15(3):191-5.

36 Galili Y, Lytle M, Bartolomei J, Amandeep K, Allen N, Carlan SJ, et al. Clear-Cell Carcinoma of the Ovary with Bilateral Breast Metastases. Case Rep Oncol Med. 2019;2019:8013913.

\section{Karger'k}

